\title{
Perioperative Management of Obstructive Sleep Apnea
}

\author{
Roop Kaw ${ }^{1} \cdot$ Peter C. Gay ${ }^{2}$
}

Published online: 12 October 2015

(C) Springer International Publishing AG 2015

\begin{abstract}
Numerous studies have now described complications in patients with obstructive sleep apnea (OSA) associated with various types of surgical encounters both from the standpoint of anatomical location and timing of the surgery such as inpatient or outpatient. Cardiac, pulmonary, and cognitive events are the best studied but are sometimes ill-defined and difficult to equate between different investigators such that the data may appear conflicting and provides challenges to guideline development and management. This has resulted in variant national- and local-level protocols to address risk factor assessment and perioperative practice patterns. What seems to be clear is that increased postoperative events do occur in patients with OSA, more so in the presence of obesity hypoventilation, but the incidence of true mortality and serious morbidity seems to be low such that these outcomes remain the most poorly understood albeit still of significant interest. In the following review, we hope to present a balanced discussion of these issues to foster broader understanding and acceptance of the concerns arising from the perioperative management of patients with OSA and encourage further research to provide direction into the development of more uniform appropriate guidelines.
\end{abstract}

This article is part of the Topical Collection on Sleep Related Breathing Disorders

Peter C. Gay

pgay@mayo.edu

Roop Kaw

KAWR@ccf.org

1 Cleveland Clinic, Cleveland, OH, USA

2 Mayo Clinic, Rochester, MN, USA
Keywords Sleep apnea $\cdot$ Sleep · Obstructive sleep apnea Perioperative practice $\cdot$ OSA

\section{Introduction}

Our primary goal is to first identify the reported postoperative complications of obstructive sleep apnea (OSA) patients for which we can provide the most acceptable data. For a pulmonary event, this would include respiratory failure and unanticipated transfer to the ICU and re-intubation. For cardiac issues, this would include cardiac arrhythmias and infarct/CHF events, and for other concerns, we will discuss delirium, extended length of stay, ICU use, mortality, and various morbidities. Once identified, the obvious next effort should be directed at risk reduction in both the pre- and intraoperative environments and will be next discussed. With ever-enhancing technologic capabilities for early detection of clinical compromise, the decisions regarding the appropriate level of monitoring to prevent complications without undue expenditure of resources must also be carefully considered.

\section{Postoperative Complications in Patients with OSA}

\section{Postoperative Respiratory Failure in OSA}

Numerous studies have reported respiratory complications after non-cardiac surgery in patients with OSA. Among these, postoperative respiratory failure can be the most worrisome, although not consistently reported, depending upon the definition used for respiratory failure among the different studies as well as their respective sample sizes. In the largest observational study to date, Memtsoudis et al. reported a fivefold increase in intubation and mechanical ventilation after 
orthopedic surgery in OSA patients $(n \sim 66,000)$ and twofold increase after general surgery $(n \sim 48,000)$ among patients with OSA (identified by ICD-9 diagnosis) compared to controls [1]. Another study using the same database showed that the incidence for repeat emergent intubation and mechanical ventilation was highest in the first $24 \mathrm{~h}$ and no difference was noted across different types of surgical categories [2]. However, interestingly enough after adjusting for age, sex, and Charleston co-morbidity index, patients without sleep disordered breathing (SDB) requiring emergent intubation had significantly worse outcomes compared to those with SDB requiring emergent intubation. The authors attribute this finding to potentially rapidly reversible upper airway obstruction in the setting of opioid and sedative use in the immediate postoperative period or perhaps a lower threshold used for re-intubation or ICU transfer. Both of these studies used administrative data from the National Information System (NIS) database; the sample was dominated by the orthopedic surgical cohort ( $n \sim 2.6$ million) and showed a steadily increasing prevalence of OSA over the duration of the study period. A meta-analysis of 3942 patients with OSA, a majority of whom were confirmed by polysomnography, confirmed higher incidence of respiratory failure compared to patients without OSA (OR, 2.43; $p=0.003$ ) [3]. More recently, Mutter et al. compared $>2500$ patients with OSA, half of them undiagnosed at the time of surgery, to 16,000 controls in the Manitoban health administrative database and confirmed higher postoperative respiratory complications regardless of early diagnosis and prescription of continuous positive airway pressure (CPAP) [4]. Early reports among patients with obesity hypoventilation syndrome (OHS) undergoing elective surgery show that the incidence of postoperative respiratory failure may be even higher and that OHS is more likely to be unrecognized before elective non-cardiac surgery when compared to OSA [5].

\section{Unanticipated ICU Transfer After Surgery}

Unanticipated ICU transfer after surgery has often been used as an outcome measure among patients with OSA. This measure is, however, not always so easy to track as patients can often be sent to ICU for overnight observation. If the observation transfers to ICU are excluded [6], the presence of OSA appears to be significantly associated with higher odds of ICU transfer (OR $2.29 ; 95 \%$ CI $1.62-3.24, p \leq 0.00001, I^{2}=57$ to $68 \%, p \leq 0.02$ ) after surgery. A recent study using the STOP-BANG score as a predictor of postoperative critical care admission reported OR of 2.2 (95 \% CI: 1.1-4.6; $p=0.037$ ), 3.2 (95\% CI: 1.2-8.1; $p=$ 0.017 ), and 5.1 (95\% CI: $1.8-14.9 ; p=0.002$ ) for STOPBANG scores of 4,5 , and $\geq 6$, respectively [7].

\section{Cardiovascular Complications}

A higher incidence of postoperative cardiovascular events was reported in a meta-analysis, but limited specific cardiac complications had been separately looked at in the literature until recently [3]. Mokhlesi et al. reported a higher incidence of atrial fibrillation after orthopedic and prostate surgery in patients with OSA, but it is not clear if this was a new onset atrial fibrillation or chronic atrial fibrillation diagnosed for the first time in the perioperative period [2]. Recently, however, OSA has been shown to be a strong predictor of new onset atrial fibrillation after $\mathrm{CABG}$, which in turn led to a higher postoperative length of stay [8]. In the largest administrative cohort of postoperative outcomes to date, comparing treated and untreated patients with OSA, higher rates of cardiac arrest and shock were noted in patients in the undiagnosed group [4].

\section{Postoperative Delirium}

A higher incidence of postoperative encephalopathy was reported in a sample of 37 patients with apnea-hypopnea index (AHI) $\geq 5$ undergoing elective cardiac surgery compared to 185 propensity-matched controls [9]. History of OSAS has been associated with postoperative delirium after knee replacement [10]. More recently, in a prospective cohort study of 92 patients undergoing elective cardiac surgery with extracorporeal circulation, a median $\mathrm{AHI} \geq 19$ was associated with an almost sixfold increased risk of postoperative delirium (OR 6.4; $95 \%$ confidence interval: $2.6-15.4 ; s<0.001)$ [11]. No significant differences were noted in the mean oxygen saturation or the mean proportion of time with oxygen saturation $<90 \%$, between patients with and without postoperative delirium.

\section{Postoperative Mortality}

In 1997, Ostermeier et al. reported three deaths from sudden postoperative respiratory arrest associated with epidural opioids in patients with sleep apnea [12]. Sudden cardiac death (SCD) is more likely to occur during the sleep hours (12 midnight to 6:00 a.m.) among patients with OSA compared to those without OSA (50\% compared to $21 \%$; RR: 2.57 ) [13]. Recently, the same group of authors showed that among patients with OSA, the lowest nocturnal oxygen desaturation and AHI were independent predictors of SCD [14]. Few studies that have looked at postoperative mortality did not find any association between OSA and mortality after elective surgery $[2,15]$. This observed lack of association between postoperative mortality and OSA does not necessarily mean that death cannot be an expected sentinel event in a postoperative patient with OSA especially exposed to additional insults like failed airway, unmonitored respiratory depression from opioid, and/ or sedative administration especially with PCA infusions and others. Clearly, the incidence of such events in the 
perioperative setting when reported from the large administrative databases is low enough to stand the test of statistical rigor of association.

\section{Perioperative Risk Reduction Strategies in OSA}

\section{Preoperative Management}

Reductions in pharyngeal dilator muscle activation and lung volume [16], delayed (average 100-200 ms) or abolished upper airway muscle tone during inspiration [17, 18], impaired upper airway neuromotor responses, and variability in the collapsibility of the upper airway [19] can predispose patients with OSA to a variety of airway management issues particularly when anesthesia and opioids come into play.

Patients with known OSA using CPAP at home should be advised to bring their machine to the hospital at the preoperative visit. Patients who are non-compliant should be encouraged to use it more consistently both preoperatively and postoperatively.

\section{Difficult Intubation}

Patients with OSA often present with difficulty at intubation (DI). Kheterpal et al. reported identical predictors like body mass index $(\mathrm{BMI})>30 \mathrm{Kg} / \mathrm{m}^{2}$ and neck circumference $>40 \mathrm{~cm}$ for both OSA and DI among a cohort of 50,000 anesthetized patients [20]. The same can be said of the other clinical predictors of DI like the Mallampati score and short thyromental distance span [21]. When using general anesthesia, the possibility of difficult intubation should be kept in mind and preparation for induction and intubation should follow the ASA difficult airway guidelines [22, 23]. Passive closing pressures (PCLOSE) among anesthetized patients with OSA can be up to $9 \mathrm{~cm} \mathrm{H}_{2} \mathrm{O}$ higher than those among patients without OSA patients and can be lowered by $6 \mathrm{~cm} \mathrm{H}_{2} \mathrm{O}$ by proper positioning of the patient in the sitting position. Passive closing pressures can decrease by $4 \mathrm{~cm} \mathrm{H}_{2} \mathrm{O}$ in the sniffing or the ramp position thus allowing both tracheal intubation and ventilation [24]. Awake intubation (with regional anesthesia and or sedation) and rapid induction are preferred over slow induction when DI is suspected [25]. Pharyngeal airway closure can be prevented by application of $10 \mathrm{~cm}$ of positive end expiratory pressure (PEEP) during positive pressure ventilation in even among the most severe cases of OSA.

\section{Intraoperative Management}

In patients with previously undiagnosed OSA and high clinical suspicion of OSA based on preoperative assessment, if significant use of opioids and sedation is anticipated based on the type of the surgical procedure, consideration should be given to inpatient surgery as opposed to ambulatory surgery. Wherever possible, regional anesthesia and blocks are preferred, and for general anesthesia, short acting agents like desflurane and propofol are recommended. A recent study on sleep apnea patients undergoing total joint arthroplasty found that patients undergoing surgery under neuraxial anesthesia had significantly lower rates of major complications compared in which combined neuraxial and general or general anesthesia alone were used [26]. The ASA recommends general anesthesia with a secure airway over sedation without an airway particularly for procedures that may mechanically compromise the airway. If moderate sedation is used, ventilation should be continuously monitored by capnography or other automated method. Full reversal of neuromuscular blockade (when applicable) with use of a nerve stimulator should be verified prior to extubation [27]. These patients must be fully awake, normothermic, hemodynamically stable, and spontaneously breathing with and adequate respiratory rate and tidal volume prior to breathing.

Multimodal analgesia involving the use of NSAIDS, acetaminophen, tramadol, ketamine, pregabalin, and COX-2 inhibitors should be considered to minimize the use of opioids in the postoperative setting [28]. OSA patients receiving opioids are more likely than patients receiving non-opioid analgesia to have perioperative oxygen desaturations [29]. Cautious use of supplemental oxygen is advised after extubation until the patient is able to maintain his/her baseline oxygen saturation on room air.

\section{Perioperative Monitoring Protocols}

Currently, there are no data to support continuous monitoring with pulse oximetry in the postoperative period. A few institutions have developed protocols to standardize their approach to minimize risk in patients considered high risk for perioperative complications. At one institution, a clinical practice initiative was developed, utilizing an existing tool to screen patients (the Sleep Apnea Clinical Score: SACS) and a postanesthesia care unit (PACU) assessment looking for recurrent episodes of (1) apnea, (2) bradypnea, (3) desaturation, and (4) pain-sedation mismatch [30]. This allowed them to identify patients at higher risk of postoperative respiratory complications with both tools, with the highest sensitivity (odds ratio near 20) utilizing both the preoperative and postoperative evaluations. The PACU respiratory assessment was made part of the electronic charting system to ensure this was done for all patients. Patients who are high risk by preoperative screening or PACU criteria receive remote oximetry for $24-48 \mathrm{~h}$ postoperatively. If positive airway pressure (PAP) is necessary, patients go to a higher level of care, a step-down unit or an intensive care unit. Patients who require PAP postoperatively are presumed to have undiagnosed OSA and seen by a sleep consultation service prior to discharge allowing planning for 
management after discharge and follow-up recommendations. Attempts have been made to provide "just in time" hospital introduction of auto-positive airway pressure (APAP) use in undiagnosed patients who prove to be at high risk for OSA based on a previously verified (SACS) questionnaire.

Yet another major tertiary care institution arranges singlechannel home testing (oximetry) for those on CPAP to confirm the accuracy of current CPAP settings prior to surgery; whereas OSA patients with $\mathrm{SpO}_{2}<90 \%$ for $<10 \%$ of the time and CPAP compliance $<4 \mathrm{~h} /$ night were offered repeat testing with full polysomnography. For those with suspected OSA, full home portable testing (four channels) is performed within 5 days of surgery. Patients are encouraged to bring their home masks to ensure best fit although they ensure a variety of masks are available including nasal pillows, nasal masks, and full face masks. For hospital PAP devices, they chose to obtain bi-level auto devices, as these allowed them to use many modes which would be available in that device (CPAP, bi-level (S), auto bi-level). The group adopted their own standard for auto settings (EPAP $\min =5$, IPAP $\max =15$, PS $\max =4$ ) to allow physicians unfamiliar with the technology to have a credible starting place. IPAP max is kept low to reduce the risk of (1) central apnea, (2) runaway pressure due to mask leak, (3) aerophagia, and (4) pressure intolerance. Hospital monitoring of PAP therapy including telemetry pulse oximetry as well as PAP downloads are made available in real time through the computer network, facilitating sleep consultation when needed.

\section{Perioperative Intervention Protocols}

\section{Use of Continuous Positive Airway Pressure (CPAP) Therapy}

The role of CPAP used perioperatively among patients with OSA has not been fully clarified. Indirect evidence can be obtained from patients undergoing bariatric surgery who happen to have higher prevalence of OSA and have often had the chance to have a treatment plan initiated before surgery. Although some series have reported higher postoperative complication rates among bariatric surgical patients with OSA, other studies had most patients diagnosed preoperatively and treated with PAP therapy; the presence or severity of OSA did not lead to a higher postoperative complication rate [31]. Among patients with OSA cardioverted for atrial fibrillation, those treated with CPAP were less likely to develop recurrent atrial fibrillation within 12 months of cardioversion compared to patients without treatment with CPAP [32]. A recent study randomized patients with high Sleep Apnea Clinical Score (SACS) undergoing elective orthopedic surgery to standard care plus auto-PAP and to standard care alone. No differences in length of stay $(p=0.65)$ or secondary outcomes (including unplanned ICU transfer, arrhythmia, MI, or delirium) were observed between the two groups [33]. Some of the main limitations of this study include the incomplete resolution of OSA as suggested by a median residual $\mathrm{AHI}$ of 13.5 events/h, the inability to distinguish central sleep apnea from OSA, and the limited adherence to therapy in a hospital setting. Conclusive evidence mandating empiric use of CPAP perioperatively among patients not currently using it is still lacking. More studies are needed.

\section{Use of Non-Invasive Positive Pressure Ventilation (NIPPV) Therapy}

If frequent or severe airway obstruction or hypoxemia is noted in the postoperative monitoring period, consideration should be made to initiate non-invasive positive pressure ventilation (NIPPV). The primary urgency for adding NIPPV should certainly be for overlap syndrome (COPD and OSA) or patients with overt obesity hypoventilation syndrome with worsening hypercapnia. Several studies support this necessity for NIPPV over other forms of PAP therapy, and this has become the standard of practice in many areas for acute hypercapnic respiratory failure [34].

The best studied postoperative use of NIPPV in patients with obesity and sleep disordered breathing is in the bariatric surgery population [35]. Forty morbidly obese patients with known OSA undergoing laparoscopic bariatric surgery were randomly assigned to receive NIPPV immediately after tracheal extubation or supplemental oxygen and CPAP with the purpose of determining whether NIPPV affects lung function 1 day postoperatively. The forced expiratory volume in $1 \mathrm{~s}$ (FEV1), forced vital capacity (FVC), and peak expiratory flow rate were all significantly reduced in both groups from perioperative values throughout the study. At $24 \mathrm{~h}$, the NIPPV group had less reduction in the $\mathrm{FVC}(0.7 \mathrm{~L}$ versus $1.3 \mathrm{~L} ; p=$ $0.0005)$ compared to the other intervention group. This led the authors to conclude that NIPPV started immediately after extubation significantly improves lung volume at $1 \mathrm{~h}$ and 1 day postoperatively, compared with continuous positive airway pressure started in the PACU, in morbidly obese patients with OSA after bariatric surgery.

\section{Encouragement of Patient Self-Management}

One less explored avenue in this arena is now very popular in the area of blood pressure, blood glucose, and lipid management. Patients are not only expected to understand the details of their laboratory test but to make active management decisions in concert with their treating clinicians. One such example has been trialed in the area of post-cardiac surgery patients who are empowered with a high level or understanding and participation in their expected progress [36]. Most patients were able to work through over 50 care modules over 5 to 
7 days and completed more than $85 \%$ of them before they left the hospital. Patients did best with the self-assessment tools, completing more than $97 \%$ of them, and the majority returned a post-discharge survey with $>90 \%$ expressing overall satisfaction with their care and felt they were well informed about their care while in the hospital. Patients felt comfortable using the myCare program within a day or two of use, and nearly $100 \%$ felt better prepared to manage their own care after they left the hospital. This type of approach and preliminary data encourages the use of patient education and participation through the myCare program which may help shorten length of stay, reduce cost of care, and improve patient independence after leaving the hospital.

\section{Conclusions}

Clinicians taking care of patients with OSA are often intrigued and concerned about safe perioperative care. Studies to date have shown that OSA is vastly underestimated in the general population, often undiagnosed in the surgical patient, and hence can lead to higher postoperative complications, but the risk of severe morbidity and mortality appears low such that skepticism and complacency about management plans are pervasive. Early clinical recognition of OSA should help in designing a safer care plan. Several perioperative interventional management practices could be employed to both prevent and treat complications of surgery in OSA patients, but there is a wide avenue of research worthy of further exploration with a focus on hard meaningful outcomes.

\section{Compliance with Ethical Standards}

Conflict of Interest Roop Kaw and Peter C Gay declare that they have no conflict of interest.

Human and Animal Rights and Informed Consent This article does not contain any studies with human or animal subjects performed by any of the authors.

\section{References}

1. Memtsoudis S, Liu SS, Ma Y, et al. Perioperative pulmonary outcomes in patients with sleep apnea after non-cardiac surgery. Anesth Analg. 2011;112:113-21.

2. Mokhlesi B, Hovda MD, Vekhter B, Arora VM, Chung F, Meltzer DO. Sleep-disordered breathing and postoperative outcomes after elective surgery: analysis of the nationwide inpatient sample. Chest 2013;12-2905.

3. Kaw R, Chung F, Pasupuleti V, Mehta J, Gay PC, Hernandez AV. Meta-analysis of the association between obstructive sleep apnoea and postoperative outcome. Br J Anaesth. 2012;109:897-906.
4. Mutter TC, Chateau D, Moffatt M, Ramsey C, Roos LL, Kryger M. A matched cohort study of postoperative outcomes in obstructive sleep apnea: could preoperative treatment prevent complications? Anesthesiology. 2014;121(4):707-18.

5. Kaw R, Bhateja P, Paz y Mar H, Hernandez AV, Ramaswamy A, Deshpande A, Aboussouan LS. Postoperative complications in patients with obesity hypoventilation syndrome undergoing elective non-cardiac surgery. Chest doi: 10.1378/chest.14-3216.

6. Liao P, Yegneswaran B, Vairavanathan S, Zilberman P, Chung F. Postoperative complications in patients with obstructive sleep apnea: a retrospective cohort matched study. Can J Anaesth. 2009;56(11):819-28.

7. Chia P, Seet E, Macachor JD, Iyer US, Wu D. The association of preoperative STOP-BANG scores with postoperative critical care admission. Anesthesia. 2013;68(9):950-2.

8. EM V o, Hamilton A, Petsikas D, Payne D, Redfearn DP, Zhang S, et al. Effect of preoperative obstructive sleep apnea on the frequency of atrial fibrillation after coronary artery bypass grafting. Am J Cardiol. 2014;113(6):919-23.

9. Kaw R, Golish J, Ghamande S, Burgess R, Foldvary N, Walker E. Incremental risk of obstructive sleep apnea on cardiac surgical outcomes. J Cardiovasc Surg (Torino). 2006;47:683-9.

10. Flink BJ, Rivelli SK, Cox EA, White WD, Falcone G, Vail TP, et al. Obstructive sleep apnea and incidence of postoperative delirium after elective knee replacement in the non-demented elderly. Anesthesiology. 2012;116:788-96.

11. Roggenbach J, Klamann M, von Haken R, Bruckner T, Karck M, Hofer S. Sleep-disordered breathing is a risk factor for delirium after cardiac surgery: a prospective cohort study.

12. Ostermeier AM, Roizen MF, Hautkappe M, Klock PA, Klafta JM. Three sudden postoperative respiratory arrests associated with epidural opioids in patients with sleep apnea. Anesth Analg. 1997;85: 452-6.

13. Gami AS, Howard DE, Olson EJ, Somers VK. Day night pattern of sudden death in obstructive sleep apnea. N Engl J Med. 2005;352(12):1206-14.

14. Gami AS, Olson EJ, Shen WK, Wright RS, Ballman KV, Hodge DO, et al. Obstructive sleep apnea and the risk of sudden cardiac death: a longitudinal study of 10,701 adults. J Am Coll Cardiol. 2013;62(7):610-8. doi:10.1016/j.jacc 2013.04.080.

15. Lockhart EM, Willingham MD, Abdallah AB, Helsten DL, Bedair BA, Thomas J, et al. Obstructive sleep apnea screening and postoperative mortality in a large surgical cohort. Sleep Med. 2013;14(5):407-15.

16. Hillman DR, Platt PR, Eastwood PR. Anesthesia, sleep, and upper airway collapsibility. Anesthesiol Clin. 2010;28:443-55.

17. Remmers JE, deGroot WJ, Sauerland EK, et al. Pathogenesis of upper airway occlusion during sleep. J Appl Physiol. 1978;44: 931-8.

18. Strohl KP, Hensley MJ, Hallett M, et al. Activation of upper airway muscles before onset of inspiration in normal humans. J Appl Physiol. 1980;49:638-42.

19. McGinley BM, Schwartz AR, Schneider H, Kirkness JP, Smith P, Patil SP. Upper airway neuromuscular compensation during sleep is defective in obstructive sleep apnea. J Appl Physiol. 2008;105:197205.

20. Kheterpal S, Martin L, Shanks AM, et al. Prediction and outcomes of impossible mask ventilation: a review of 50,000 anesthetics. Anesthesiology. 2009;110:891-7.

21. Shiga T, Wajima Z, Inoue T, Sakamoto A. Predicting difficult intubation in apparently normal patients: a meta-analysis of bedside screening test performance. Anesthesiology. 2005;103:429-37.

22. Hiremath AS, Hillman DR, James AL, et al. Relationship between difficult tracheal intubation and obstructive sleep apnoea. Br J Anaesth. 1998;80:606-11. 
23. Rosenblatt WH, Whipple J. The difficult airway algorithm of the American Society of Anesthesiologists. Anesth Analg. 2003;96: 1233.

24. Isono S. Optimal combination of head, mandible and body positions for pharyngeal airway maintenance during perioperative period: lesson from pharyngeal closing pressures. Semin Anesth. 2007;26:83-93.

25. Benumof JL. Management of the difficult airway. With special emphasis on awake intubation. Anesthesiology. 1991;75:1087110.

26. Memtsoudis SG, Stundner O, Rasul R, et al. Sleep apnea and total joint arthroplasty under various types of anesthesia: a populationbased study of perioperative outcomes. Reg Anesth Pain Med. 2013;38:274-81.

27. Murphy GS, Szokol JW, Marymont JH, Greenberg SB, Avram MJ, Vender JS. Residual neuromuscular blockade and critical respiratory events in the postanesthesia care unit. Anesth Analg. 2008;107: $130-7$.

28. Chung SA, Yuan H, Chung F. A systematic review of obstructive sleep apnea and its implications for anesthesiologists. Anesth Analg. 2008;107:1543-63.

29. Bolden N, Smith CE, Auckley D, Makarski J, Avula R. Perioperative complications during use of an obstructive sleep apnea protocol following surgery and anesthesia. Anesth Analg. 2007;105:1869-70.
30. Gali B, Whalen FX, Gay PC, et al. Management plan to reduce risks in perioperative care of patients with presumed obstructive sleep apnea syndrome. J Clin Sleep Med. 2007;3:582-8.

31. Weingarten TN, Flores AS, McKenzie JA, et al. Obstructive sleep apnoea and perioperative complications in bariatric patients. $\mathrm{Br} \mathrm{J}$ Anaesth. 2011;106:131-9.

32. Kanagala R, Murali NS, Friedman PA, et al. Obstructive sleep apnea and the recurrence of atrial fibrillation. Circulation. 2003;107: 2589-94.

33. O'Gorman SM, Gay PC, Morgenthaler TI. Does auto-titrating positive airway pressure therapy improve postoperative outcome in patients at risk for obstructive sleep apnea syndrome?: A randomized controlled clinical trial. Chest. 2013;144:72-8.

34. Caples SM, Gay PC. Noninvasive positive pressure ventilation in the intensive care unit: a concise review. Crit Care Med. 2005;33(11):2651-8.

35. Neligan PJ, Malhotra G, Fraser M, et al. Noninvasive ventilation immediately after extubation improves lung function in morbidly obese patients with obstructive sleep apnea undergoing laparoscopic bariatric surgery. Anesth Analg. 2010;110(5):1360-5.

36. Cook DJ, Pulido JN, Thompson JE, Dearani JA, Ritter MJ, Hanson $\mathrm{AC}$, et al. Standardized practice design with electronic support mechanisms for surgical process improvement: reducing mechanical ventilation time. Ann Surg. 2014;260(6):1011-5. 